\title{
COSMIC RAYS AND RADIO WAVES AS MANIFESTATIONS OF A HOT UNIVERSE
}

\author{
T. GOLD \\ Harvard College Observatory, Cambridge, Massachusetts, U.S.A. \\ F. HOYLE \\ University of Cambridge, England
}

Attempts to explain both the expansion of the universe and the condensation of galaxies must be very largely contradictory so long as gravitation is the only force field under consideration. For if the expansive kinetic energy of matter is adequate to give universal expansion against the gravitational field it is adequate to prevent local condensation under gravity, and vice versa. This is why, essentially, the formation of galaxies is passed over with little comment in most systems of cosmology. Yet the galaxies, and the clusters in which they are often found, are such an important characteristic property of the universe that it is unsatisfactory to dismiss their origin in the vague term "fluctuation phenomenon."

The situation is much improved by introducing electromagnetic forces. In particular it is attractive to regard the galaxies as regions of local cooling in a hot gas. Condensation is not then primarily a gravitational process, but a result of pressure in the external hot gas acting at the surface of a cooled region. After compression, once higher densities are attained, gravitation can of course become the dominating force, but only after the magnitude of the gravitational force has been increased by a factor $\sim 10^{2}$.

Suppose cooling takes place in such a way that the internal pressure is comparable with the external pressure. Then we have

$$
n T=\text { constant }
$$

throughout the condensation process, where $n$ is the number density of atoms (essentially hydrogen), and $T$ is the temperature, both these quantities being taken within the condensation.

Typical values at the end of the condensation process are $n \cong 1$ atom $/ \mathrm{cm}^{3}$, $T \cong 10^{4} \mathrm{~K}$, corresponding to a pressure of about $10^{-12}$ dynes $/ \mathrm{cm}^{2}$. This requires the constant in the above equation to be $\sim 10^{4}$. Writing $n_{0}, T_{0}$ for initial values, the condition of approximately equal internal and external pressure therefore requires

$$
n_{0} T_{0}=10^{4}
$$

to be satisfied by the hot gas in which the condensation forms.

If, further, we require the hot gas to be able to cool in a time scale of cosmical order, i.e. $\frac{1}{3} H^{-1}$, where $H^{-1}$ is the reciprocal of the Hubble constant 583 
$\left(4 \times 10^{17}\right.$ seconds), a second relation between $n_{0}$ and $T_{0}$ can be obtained. Employing this second relation gives $n_{0} \cong 10^{-3}$ atoms $/ \mathrm{cm}^{3}, T_{0} \cong 10^{7} \mathrm{~K}$.

The rate of radiation by ionized hydrogen of temperature $T_{0}$ and number density $n_{0} / \mathrm{cm}^{3}$ is

$$
8.7 \times 10^{-4} T_{0}^{\frac{1}{2}} n_{0}\left(1+\frac{3.85 \times 10^{5}}{T_{0}}\right) \text { ergs/gram second. }
$$

The thermal energy possessed by the hydrogen is

$$
\left(1.3 \times 10^{13}+2.5 \times 10^{8} T_{0}\right) \text { ergs/gram. }
$$

We simply require that a gas radiating at a rate given by (3) for a time $\frac{1}{8} H^{-1}$ shall be able to dispose of the amount of energy given by (4).

The behavior of a condensation with these initial values of $n_{0}, T_{0}$ is shown in Fig. 1, it being assumed that (1) remains satisfied throughout the cooling process. The startling point emerges that after 90 per cent of the total cooling time the condensation has only shrunk to about two-thirds of its initial diameter. After 99 per cent of the cooling time the condensation has shrunk to only a little less than one-half of its initial diameter. Clearly the final stages of condensation are quite catastrophic, the reason lying in the rapid acceleration of the cooling process.

This type of behavior is exactly what is required to explain the condensation of a very large cloud into a number of galaxies rather than into one single compact mass, since those places in the cloud where cooling is initially a little more rapid than elsewhere will reach the point of catastrophic instability before the density throughout the main mass has increased by any large factor.

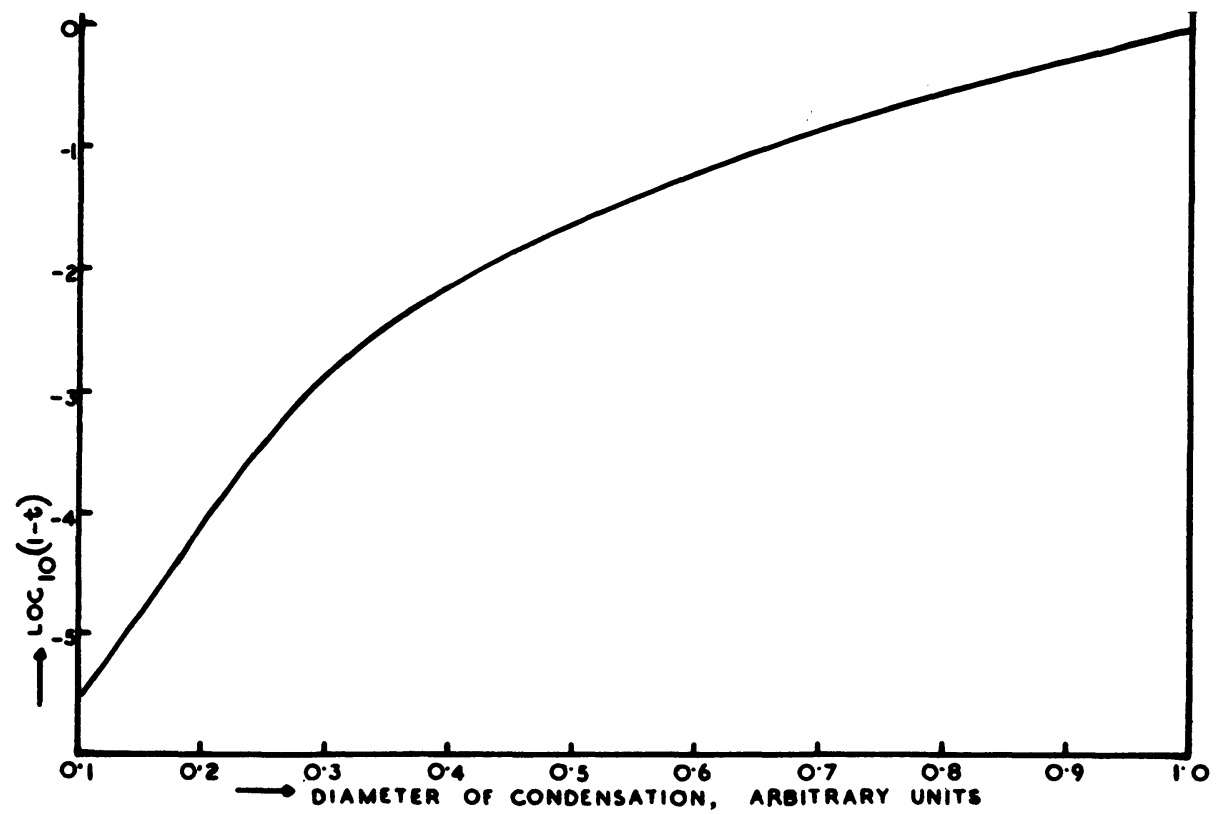

FIG. 1. Formation of a galaxy by cooling. Temperature $10^{7}{ }^{\circ} \mathrm{K}$, density $10^{-27} \mathrm{grams} / \mathrm{cm}^{3}$, unit of time $1 / 3 H$. 
The later stages of cooling are indeed so very rapid that it seems doubtful if a cooled blob of matter can be compressed by surrounding material fast enough to maintain the internal pressure at a value even approximately equal to the external pressure. This situation occurs when the diameter of the blob has declined to about 40 per cent of its initial value. Thereafter the blob is driven inward by a gross difference of internal and external pressure.

It is to be expected that turbulent motions will then be developed within such a blob, the speed of the motions being comparable with, but somewhat less than, the velocity of sound in the hot surrounding gas. The latter's temperature $\sim 10^{7}{ }^{\circ} \mathrm{K}$ gives turbulent motions $\sim 250 \mathrm{~km} / \mathrm{second}$.

It seems significant that the rotary motions of the galaxies are of this order, as indeed are the random motions of the galaxies relative to the general cosmical background. It is also significant that in order for the self-gravitation of a condensed blob to be able to control motions $\sim 250 \mathrm{~km} / \mathrm{second}$, the mass of the blob must be $\sim 10^{11} \odot$, or more. Condensations with smaller mass would simply fly into a number of fragments. These considerations therefore suggest a natural separation of dwarf galaxies (small fragments) from the standard class of large galaxy, the latter having masses in the range $10^{11}$ to $10^{12} \odot$.

It may be mentioned here that $Z$ wicky and others have observed a structure that requires an explanation. In several galaxies long "tails" are seen as low-luminosity curved streaks stretching out many galactic diameters. Frequently they make a connection between neighboring galaxies.

This phenomenon is of particular importance because of the difficulty of finding any explanation for it within the usual ideas. A tidal perturbation of a galaxy can alter its shape, but cannot draw out a long narrow filament. Magnetohydrodynamic processes could be responsible if the material were gaseous, but then, lacking appreciable self-gravitation, the filament would rapidly disperse in the case of a low-pressure, low-temperature universe. So, although little is known about the "tails," we are forced to think of some agency that can maintain these remarkable configurations for the time necessary for them to be drawn out and that can supply the energy necessary to make them shine.

In the case of a hot universe there is a clear interpretation. Any gaseous filament drawn out, or produced as the wake of a galaxy in the intergalactic medium, will now not only fail to disperse, but can even grow denser. The pressure of the hot surrounding gas will push fresh material onto it which will be cooled by contact with the material of the filament. Stars may then form, and excitation of the gas can result from energy liberated at the interface between the hot and the cold gas.

These considerations seem so encouraging that it is worth while to follow the argument further, even though it leads to somewhat remarkable conclusions. In the first place the value $n_{0} \sim 10^{-3}$ atoms $/ \mathrm{cm}^{3}$ does not correspond to the present mean density of matter in space, which can scarcely be much higher than $10^{-5}$ atoms $/ \mathrm{cm}^{3}$. Radiative cooling at the latter density is small. A continuation of the argument therefore requires us to follow one or the other of the following lines: 
(a) The galaxies condensed at an earlier phase of the expanding universe at a time when the mean density of matter in space was of order $10^{-3}$ atoms $/ \mathrm{cm}^{3}$.

(b) The radiative cooling considered above is merely a secondary process following after a quite different primary cooling that occurs in cosmological material of density $10^{-6}$ atoms $/ \mathrm{cm}^{3}$.

The alternative $(a)$ cannot be followed in the steady-state cosmological theory, since in this theory the density was never greater than its present value. On the other hand, it seems impossible to obtain high temperatures in relativistic cosmology, since adiabatic expansion from an initially very high density reduces the temperature to a value of order $1^{\circ} \mathrm{K}$. For these reasons alternative $(a)$ seems unattractive. Moreover, the high-temperature requirement suggests that we should follow alternative $(b)$ within the framework of the steady-state theory.

An interesting feature appears immediately. Again requiring an equality of pressure everywhere throughout space, equation (1) gives $T \sim 10^{\circ}{ }^{\circ} \mathrm{K}$ when $n \sim 10^{-5}$ atoms $/ \mathrm{cm}^{3}$. Such a temperature corresponds to a thermal energy of $\sim 2.5 \times 10^{17} \mathrm{ergs} / \mathrm{gram}$ of material, which is precisely the energy yielded by neutron decay (after allowing for the loss of the energy carried away by neutrinos). Thus if the created matter of the steady-state theory is taken to be initially in the form of neutrons, the resulting temperature of the general cosmological material is exactly of the required order, viz. $10^{\circ}{ }^{\circ} \mathrm{K}$.

The radiation by bremsstrahlung from such a hot gas at very low density is small, and no immediately obvious effects can be suggested. The radiation is in a spectral range that is rapidly absorbed by the carbon, oxygen, nitrogen, and neon in a galaxy, so that its effects could be expected to show only in a thin outer layer around a galaxy where secondary radiation may be produced.

We therefore arrive at a picture of the galaxies with internal temperatures $\sim 10^{4} \mathrm{~K}$ (or less when dust is formed) immersed in a medium of temperature $\sim 10^{7} \mathrm{~K}$, and of the latter medium itself being immersed in the general cosmological material at temperature $\sim 10^{\circ} \mathrm{K}$. It is then thermodynamically possible for highly efficient heat engines to work between the cosmological material and the cooler denser material immersed within it. The essential feature of such an engine is that almost the whole of the energy of the very hot cosmological material is converted into mechanical motion; radiative dissipation, therefore, need only be very small. Our suggestion is that this mechanical motion provides the source of the large-scale electromagnetic effects in the universe, in particular the building of magnetic fields and the acceleration of cosmic rays. It is emphasized that this source is enormously more powerful than any galactic source can possibly be, the energy available being $\sim 10^{8}$ times greater.

This idea meets with an immediate success. The energy of the cosmological gas is $\sim 2.5 \times 10^{-12} \mathrm{ergs} / \mathrm{cm}^{3}$. If this energy is first converted to mechanical work, and subsequently to cosmic-ray energy (e.g., through the operation of a Fermi process) then the cosmic-ray energy density must be $\sim 10^{-12} \mathrm{ergs} / \mathrm{cm}^{3}$ in excellent agreement with the observed value.

It is also possible to obtain a far clearer understanding of the origin of the 
cosmic-ray energy spectrum than has ever been the case for purely local galactic theories of cosmic-ray acceleration. For a Fermi process a particular cosmic-ray particle gains energy at an average rate given by the equation

$$
\frac{d E}{d t}=r E
$$

where $\gamma$ is a constant independent of $E$, the particle energy. If all the energy of the hot cosmological gas were fed into preexisting cosmic rays (none into new cosmic rays) then $r$ turns out to be $3 H$ ( $H$ is the Hubble constant). If, on the other hand, only half the energy goes into preexisting cosmic rays, and half into newly injected cosmic rays, together with other processes (e.g., maintaining an intergalactic magnetic field), then $r=1.5 \mathrm{H}$. Although a particular particle continues to gain energy in accordance with (5), the expansion of the universe weakens the density of old particles, the weakening in the steady-state theory being proportional to $\exp (-3 H t)$, where $t$ is the time. We therefore have a gain rate exp $\gamma t$ and a loss rate $\exp (-3 H t)$. By using Fermi's argument, this leads to an energy spectrum $d E / E^{(1+3 B / \gamma)}$. With $r=$ $1.5 H$, we have $d E / E^{3}$, in agreement with the higher portions of the observed cosmic-ray spectrum.

The point of real importance is not to explain the precise form of the energy spectrum, however, but to see that its form is a genuinely universal property, both the exponential factors exp $\gamma t$ and $\exp (-3 H t)$ depending on the largescale properties of the universe, not on some purely local process. This point has already been remarked on by Farley (Varenna Conference on Cosmic Rays, 1957).

High-energy electrons are produced in nuclear collisions by the cosmic rays. Assuming the cross section to be approximately independent of the energy of the colliding particles, the energy spectrum of the secondary electrons will have a similar form to that of the cosmic rays. Since an electron-energy spectrum $d E / E^{3}$ leads to a radio-emission spectrum of the form $d \nu / \nu$, which seems to be very characteristic of nongalactic sources, it appears that the general uniformity of the latter may well be an outcome of processes that occur on a universal scale.

By taking the cross section for these nuclear collisions as $2 \times 10^{-26} \mathrm{~cm}^{-2}$, and the energy density of the cosmic rays as 1 electron volt $/ \mathrm{cm}^{3}$, it can readily be shown that under normal cosmological conditions (with a mean intergalactic density of $10^{-29} \mathrm{grams} / \mathrm{cm}^{3}$ ) the electron energy density will be $\sim 10^{-4}$ electron volts $/ \mathrm{cm}^{3}$. If, on the other hand, the local density in a particular region is $\sim 10^{-27}$ grams $/ \mathrm{cm}^{3}$, the electron-energy density may well rise to $\sim 10^{-2}$ electron volts $/ \mathrm{cm}^{3}$ in that region.

The latter situation is likely to be applicable to galaxies in, and near, large clusters. The comparatively close proximity of our own Galaxy to the Virgo Cloud makes it reasonable to suppose that our position in space is privileged in this respect, and that the electron energy density in our locality is of order $10^{-2}$ electron volts $/ \mathrm{cm}^{3}$. This is of the required magnitude to explain the general emission by the Galaxy and by its halo.

The electron-energy density must be considerably higher in the strong radio 
sources. But the magnetic intensity in these sources must also be considerably higher than its general cosmological value. Thus it is difficult to imagine that the magnetic intensity in the Cygnus A source can be much less than $10^{-6}$ gauss, whereas the general cosmological intensity is probably not greater than $10^{-7}$ gauss. The compression necessary to increase such a cosmological field to the Cygnus $A$ value would be capable of increasing the electron energy density by at least a comparable factor; an initial energy density $\sim 10^{-2}$ electron volts would be increased to a value of order 10 or even $10^{2}$ electron volts $/ \mathrm{cm}^{3}$. At a magnetic intensity $\sim 10^{-5}$ gauss, this electron energy density is adequate to give a radio emissivity per unit volume of the order found in the Cygnus source.

The origin of such a compression raises difficult and interesting questions, for it scarcely seems that so powerful a compression could arise simply from the cooling process discussed previously. It is not impossible, however, that the compression arises from an enormous intergalactic pinch phenomenon.

\section{Discussion}

Bok: It may be useful to note in connection with Dr. Gold's comments that the pair of galaxies NGC 4038/39-which are, incidentally, a strong discrete radio source-have two strong, curved, and narrow arcs protruding from the pair. We note further the presence of marked knots in the main bodies of NGC 4038/39.

Oort: I do not agree with Dr. Gold that it is so very difficult to understand the existence of bridges between galaxies without invoking a high-temperature intergalactic medium. If two galaxies that have collided contained clouds of widely varying mass, these clouds will be strung out along a straight or curved path between the two galaxies. Stars might well be formed in the colliding clouds. I do not think that one need fear that the connecting arm would have dispersed too much, for the thermal motions of the order of $10 \mathrm{~km} / \mathrm{second}$ are small compared to the probable relative velocity of the two galaxies, which may be taken to be of the order of $1000 \mathrm{~km} / \mathrm{second}$.

Margaret Burbidge: With regard to the faint extensions from galaxies ("Zwicky tails"), don't they sometimes occur from single galaxies?

Minkowski: It is not always easy to be certain which other galaxy might be involved. It is possible for a galaxy to be distorted by a very much fainter system. An example is the highly distorted object NGC 1741; the second system is a small and condensed galaxy. Seen at a larger distance, it might be difficult to recognize the smaller, fainter galaxy as the source of the distortion. In a cluster it may become impossible to single out the perturbing galaxy.

Keller: Why isn't it possible to detect the electromagnetic radiation one would expect hot intergalactic gas to emit?

Hoyle: The main radiation by the gas is in the form of quanta at about $1 \mathrm{kV} / \mathrm{s}$. These are unfortunately absorbed by the $K$-electrons of carbon, nitrogen, and oxygen in the interstellar gas. This prevents direct observation of these quanta from the vicinity of the earth. It is possible, however, that secondary exciting effects (e.g., of $\lambda$ 3727) might be observed. 